\title{
Novel Food Solutions to Diabetes and Obesity
}

\author{
Pushparajah Thavarajah* \\ Plant and Environmental Sciences, Clemson University, USA
}

Submission: October 27, 2017; Published: October 30, 2017

*Corresponding author: Pushparajah Thavarajah, Plant and Environmental Sciences, 270 Poole Agricultural Center, Clemson University SC, BOV Solutions, 1105 Garner Bagnal Blvd, Statesville, NC, USA, Email: rajah.thava@gmail.com

\begin{abstract}
Global diabetes and obesity prevalence continue to rise. There are no signs that these rising rates will come to a halt with current food production and consumption practices. Therefore, modifications to current food system are urgently needed. This article addresses the existing food problem(s), changes needed, and short-term food solutions to reduce diabetes and obesity.
\end{abstract}

\section{Introduction}

Globally, the number of people with diabetes has risen to 314 million or doubled in two decades. Among them 1.6 million die direct result of diabetes, and another 2.2 million deaths because of high blood glucose levels [1]. Although many factors responsible for diabetes, overweight and obesity are major ones. To date, worldwide obesity has tripled since 1975, and more than one third above 18 years are overweight. Among young, over 340 million children and adolescents are overweight or obese [2]. There are no signs that current diabetes and overweight/ obesity rates to decline making many more to suffer.

To solve these problems, diabetes and obesity prevention is focused on two main areas: reduce of calorie or sugar focused diets and increase in physical activity levels. Although these are essential for prevention, their impacts had been rather limited despite efforts by those program participants. Why we continue to see rise of these two epidemics? This article will briefly discuss present food production and consumption failures, and provide short term food solutions to reduce diabetes and obesity prevalence.

\section{Discussion}

The current food production, processing, and distribution is economically efficient or at its best as never seen before in human history to provide greatest caloric content for a unit cost. This system works because it continues to maximize calorie content by using sugars and oils as primary food ingredients. Those two food ingredients are nonperishable; hence, calories in them could be stored, and distributed to majority of world populations at affordable prices. In the past, calorie dense foods prevented hunger and famine. They may be still needed when natural disaster or in a military conflict prevents food supplies. However, continue to maximize only calorie comes at a greater human health costs.

To address diabetes and obesity health costs, scientific reports, interest groups, and governments time to time highlight the negative effects on sole calorie based diets. Food industry responds to these pressures, and consumer demands. Perhaps more than anything, those calorie reductions by cutting sugar and fat levels are economically favorable to produce when no other added costs to have same product profiles. This pattern of only calorie focused global food supply and demand will continue; hence, no attention to all other essential nutrients for greater human health.

Every human requires 50 plus nutrients on daily basis for healthy living. Foods that fail to provide these nutrients results in severe energy and micronutrient malnutrition. Emerging economies with larger populations (e.g. China and India) will have the greatest malnutrition burdens. Among different population groups; women and young children will face major nutritional challenges because of additional nutrient requirements for reproduction and growth. Abundance of calorie dense foods devoid any other nutrients, and continue to consume those will drive both overweigh/obesity and micronutrient malnutrition rates to much higher levels. Including the US more than one third of global population is deficient in one or more mineral and vitamin micronutrients. Micronutrient deficiency, a cause and a compounding factor, could make existing health problems worse. 
Energy and micronutrient malnutrition issues, over supply of calorie and under supply of micronutrients will continue to gain worldwide attentions because those malnutrition effects are visible. However, there is a hidden, and often neglected, perhaps most important factor in human health- gut bacteria. Simply, there are hundreds of more live bacteria than human cells in digestive tract working harder and harder. They determine how we use what we eat. Recent gut micro biome studies show their indispensable role on human health. For example, lean individuals have diverse range of bacteria to keep them lean, while obese have less diverse or relatively few species. The type and number of bacteria, their actions of what we eat, metabolites they release, and how they control what get absorbed in to the body may have roles in causing type 2 diabetes and other diseases. What are the foods that could control body weights and promote human health beneficial bacteria? Will they easily fit in to today's calorie focused food system?

Prebiotic foods, more specifically prebiotic carbohydrates, promote human health beneficial bacterial growth and their diversity. By incorporating prebiotic carbohydrates in to foods, for example resistant starch- a type of starch not breaking down to glucose by human digestive enzymes, can promote human health beneficial bacterial diversity and growth. Resistant starch is low caloric and provides the same sensory properties just like starch. Their production levels could be increased using the same cost parameters as of regular starch. They can easily replace part of starch to reduce calorie intakes, develop healthier gut bacteria, and increase micronutrients absorption. Besides resistant starches, there are other prebiotic carbohydrates: sugar alcohols, insulin and large number of low-and nondigestible carbohydrates. These carbohydrates also could provide same gut health benefits while providing sweetness to foods. Therefore, by producing these prebiotic carbohydrates in abundance, including them in commonly eaten foods, and providing at affordable prices may be food solutions to reduce diabetes and obesity.

\section{Conclusion}

Novel foods based on reduced caloric densities and prebiotic effects may be short term solution to reduce diabetes and obesity burdens. Certainly, prebiotic carbohydrates could replace most or all readily digestible carbohydrates in our foods, and may well fit in to the present food-economic system to provide greater human nutritional value.

\section{References}

1. http://www.who.int/mediacentre/factsheets/fs312/en/

2. http://www.who.int/mediacentre/factsheets/fs311/en/ 